UDC 512.5

N. Rehman, A. Z. Ansari (Aligarh Muslim Univ., India)

\title{
ON LIE IDEALS AND GENERALIZED JORDAN LEFT DERIVATIONS OF PRIME RINGS
}

\section{ІДЕАЛИ ЛІ ТА УЗАГАЛЬНЕНІ ЖОРДАНОВІ ЛІВІ ПОХІДНІ ПРОСТИХ КІЛЕЦЬ}

Let $R$ be a prime ring with characteristic different from 2 and $U$ be a Lie ideal of $R$. In this paper, we initiate the study of generalized Jordan left derivations on Lie ideals of $R$ and prove that every generalized Jordan left derivation on $U$ is a generalized left derivation on $U$. Further, it is shown that generalized Jordan left biderivation associated with the left biderivation on $U$ is either $U \subseteq Z(R)$ or a right bicentralizer on $U$.

Нехай $R$ - просте кільце 3 характеристикою, відмінною від 2, а $U$ - ідеал Лі цього кільця. У цій статті ми починаємо дослідження узагальнених жорданових лівих похідних на ідеалах Лі кільця $R$ і доводимо, що будьяка узагальнена жорданова ліва похідна на $U \in$ узагальненою лівою похідною на $U$. Крім того, встановлено, що узагальнена жорданова ліва подвійна похідна, асоційована з лівою подвійною похідною на $U, \epsilon$ або $U \subseteq Z(R)$, або правим подвійним централайзером на $U$.

1. Introduction. Throughout the present paper $R$ will denote an associative ring with center $Z(R)$. A ring $R$ is $n$-torsion free, where $n>1$ is an integer, in case $n x=0$, implies $x=0$ for all $x \in R$. As usual we write $[x, y]$ for $x y-y x$ and make use of commutator identities $[x y, z]=x[y, z]+[x, z] y$ and $[x, y z]=y[x, z]+[x, y] z$ for all $x, y, z \in R$. Recall that a ring $R$ is prime if for any $a, b \in$ $R, a R b=\{0\}$ implies that either $a=0$ or $b=0$. An additive subgroup $U$ of $R$ is said to be a Lie ideal of $R$ if $[U, R] \subseteq U$. A Lie ideal $U$ of $R$ is said to be square closed if $u^{2} \in U$ for all $u \in U$. A Lie ideal $U$ is called noncommutative if $[U, U] \neq 0$. An additive mapping $\delta: R \rightarrow R$ is called a derivation (resp. Jordan derivation) if $\delta(x y)=\delta(x) y+x \delta(y)$ (resp. $\delta\left(x^{2}\right)=\delta(x) x+x \delta(x)$ ) holds for all $x, y \in R$. An additive mapping $f: R \rightarrow R$ is called a generalized derivation if there exists a derivation $\delta: R \rightarrow R$ such that $f(x y)=f(x) y+x \delta(y)$ holds for all $x, y \in R$. An additive mapping $d: R \rightarrow R$ is said to be a left derivation (resp. Jordan left derivation) if $d(x y)=x d(y)+y d(x)$ (resp. $d\left(x^{2}\right)=2 x d(x)$ ) holds for all $x, y \in R$. Clearly, every left derivation on a ring $R$ is a Jordan left derivation but the converse need not be true in general (see, for example, [17], Example 1.1). In [3], Ashraf and the first author proved that if $R$ is a prime ring with characteristic different from 2 and $d: R \rightarrow R$ is an additive mapping such that $d\left(u^{2}\right)=2 u d(u)$ for all $u$ in a square closed Lie ideal $U$ of $R$, then $d(u v)=u d(v)+v d(u)$ for all $u, v \in U$. During the last twenty five years, there has been ongoing interest concerning the relationship between left derivation and Jordan left derivation on prime and semiprime rings (cf. $[1-4,8,9,12,16,17]$ and reference therein).

Following [18], an additive mapping $T: R \rightarrow R$ is called left (resp. right) centralizer of $R$ if $T(x y)=T(x) y(\operatorname{resp} . T(x y)=x T(y))$ holds for all $x, y \in R$. An additive mapping $T: R \rightarrow R$ is called Jordan left (resp. Jordan right) centralizer of $R$ if $T\left(x^{2}\right)=T(x) x$ (resp. $T\left(x^{2}\right)=x T(x)$ ) holds for all $x \in R$. Obviously, every left centralizer is a Jordan left centralizer. The converse is in general not true. In [18], Zalar proved that every Jordan left centralizer on a 2-torsion free semiprime ring is a left centralizer. According [2], an additive mapping $F: R \rightarrow R$ is called a generalized left derivation (resp. generalized Jordan left derivation) if there exists a Jordan left derivation $d: R \rightarrow R$ such that $F(x y)=x F(y)+y d(x)$ (resp. $F\left(x^{2}\right)=x F(x)+x d(x)$ ) holds for all $x, y \in R$. It 
is easy to see that $F: R \rightarrow R$ is a generalized left derivation if and only if $F$ is of the form of $F=d+T$, where $d$ is a left derivation and $T$ is a right centralizer on $R$. The concept of generalized left derivations cover the concept of left derivations. Moreover, a generalized left derivation with $d=0$ includes the concept of right centralizer. Since the sum of two generalized left derivations is a generalized left derivation, every map of the form $F(x)=x a+d(x)$, where $a$ is a fixed element of $R$ and $d$ is a left derivation of $R$, is a generalized left derivation. Notice that for any generalized left derivation $F$, the mapping $H: R \rightarrow R$ such that $H(x)=F(x)+x a$ or $H(x)=F(x)-x a$, where $a$ is a fixed element of $R$, is also a generalized left derivation on $R$. It is obvious to see that every generalized left derivation is a generalized Jordan left derivation. But the converse need not be true in general (see Example 1.1 of [2]). It is shown in [2] that if $R$ is a 2-torsion free prime ring, then every generalized Jordan left derivation on $R$ is generalized left derivation. Recently, Shakir [1] obtained the above mentioned result for semeprime ring. In Section 3, our objective is to prove that the conclusion of the result obtained by Ashraf and Shakir [2] remains true in the case when the ring is prime and underlying subset of $R$ is a Lie ideal.

Let $S$ be a subring of a ring $R$. A mapping $B: R \times R \rightarrow R$ is said to be symmetric if $B(x, y)=$ $=B(y, x)$ for all $x, y \in R$. Following [7], a biadditive map $B: S \times S \rightarrow R$ is called a biderivation on $S$ if it is a derivation in each argument, i.e., for each $x \in S$, the map $y \mapsto B(x, y)$ and $y \mapsto B(y, x)$ are derivations of $S$ into $R$. Typical examples are mappings of the form $(x, y) \mapsto \lambda[x, y]$, where $\lambda$ is an element of the center of $R$. The concept of biderivation was introduced by Maska [13]. Further, Bresar [7] showed that every biderivation $B$ of a noncommutative prime ring $R$ is of the form $B(x, y)=\lambda[x, y]$ for some $\lambda \in C$, the extended centroid of $R$.

According [1], a biadditive mapping $B: R \times R \rightarrow R$ is called a left biderivation (resp. Jordan left biderivation) if $B(x y, z)=x B(y, z)+y B(x, z)\left(\right.$ resp. $\left.B\left(x^{2}, z\right)=2 x B(x, z)\right)$ and $B(x, y z)=$ $=y B(x, z)+z B(x, y)\left(\operatorname{resp} . B\left(x, z^{2}\right)=2 z B(z, x)\right)$ hold for all $x, y, z \in R$. A biadditive mapping $G: R \times R \rightarrow R$ is called generalized left biderivation (resp. generalized Jordan left biderivation) if there exists a left biderivation (resp. Jordan left biderivation) $B: R \times R \rightarrow R$ such that $G(x y, z)=$ $=x G(y, z)+y B(x, z)\left(\operatorname{resp} . G\left(x^{2}, z\right)=x G(x, z)+x B(x, z)\right)$ and $G(x, y z)=y G(x, z)+z B(x, y)$ (resp. $G\left(x, z^{2}\right)=z G(x, z)+z B(x, z)$ ) hold for all $x, y, z \in R$. A biadditive map $\mathcal{T}: R \times R \rightarrow R$ is called a left (resp. right) bicentralizer if $\mathcal{T}(x y, z)=\mathcal{T}(x, z) y$ and $\mathcal{T}(x, y z)=\mathcal{T}(x, y) z$ (resp. $\mathcal{T}(x y, z)=x \mathcal{T}(y, z)$ and $\mathcal{T}(x, y z)=y \mathcal{T}(x, z))$ for all $x, y, z \in R$. A biadditive mapping $\mathcal{T}: R \times$ $\times R \rightarrow R$ is called a Jordan left (resp. right) bicentralizer if $\mathcal{T}\left(x^{2}, z\right)=\mathcal{T}(x, z) x$ and $\mathcal{T}\left(x, z^{2}\right)=$ $=\mathcal{T}(x, z) z$ (resp. $\mathcal{T}\left(x^{2}, z\right)=x \mathcal{T}(x, z)$ and $\left.\mathcal{T}\left(x, z^{2}\right)=z \mathcal{T}(x, z)\right)$ for all $x, z \in R$. The map $\mathcal{T}$ is said to be a bicentralizer if it is both a left and a right bicentralizer on $R$. This type of mappings obviously extend the concepts of centralizers. In [14], Muthana presented an algebraic study of left bicentralizers in prime rings. The last section of this paper deals with the study of generalized left biderivations of a prime ring $R$ of $\operatorname{char} R \neq 2$ under the appropriate restriction on a Lie ideal $U$ of $R$. The result of this section generalize the result obtained in [1] (Theorem 5.1).

2. Preliminaries. We collect some known results and review a few important facts about the left Martindale ring of quotients that will be needed in the subsequent discussions of a ring, $Q_{l}(R)$ will denote the left Martindale ring of quotients of a prime ring $R$. This ring was introduced by Martindale in [13] as a tool in the study of prime rings satisfying generalized polynomial identities (e.f. [6]). The center of $Q_{l}(R)$, will be denoted by $C$, and called the extended centroid of $R$. It is well known that 
$C$ is a field. Also, it is easily seen that $C$ is the centralizer of $R$ in $Q_{l}(R)$. In particular, $Z(R)=C$. The subring of $Q_{l}(R)$ generated by $R$ and $C$ is called the central closure of $R$ and will be denoted by $R_{C}$. Another subring of $Q_{l}(R)$ is $Q_{s}(R)=\left\{q \in Q_{l}(R) \mid q I \subseteq R\right.$ for some nonzero ideal $I$ of $\left.R\right\}$. This ring is known as the symmetric Martindale ring of quotients. It is easy to verify that $R \subseteq R_{C} \subseteq$ $\subseteq Q_{s}(R) \subseteq Q_{l}(R)$. Note that $a R b=\{0\}$ with $a, b \in Q_{l}(R)$ implies that $a=0$ or $b=0$. Whence we can see that $R_{C}, Q_{l}(R)$ and $Q_{s}(R)$ are prime rings.

Remark 2.1. Let $U$ be a square closed Lie ideal of $R$. Notice that $x y+y x=(x+y)^{2}-x^{2}-y^{2}$ for all $x, y \in U$. Since $x^{2} \in U$ for all $x \in U, x y+y x \in U$ for all $x, y \in U$. Hence, by definition of Lie ideal, we find that $2 x y \in U$ for all $x, y \in U$. Therefore, for all $r \in R$, we get $2 r[x, y]=$ $=2[x, r y]-2[x, r] y \in U$ and $2[x, y] r=2[x, y r]-2[y, r] \in U$, so that $2 R[U, U] \subseteq U$ and $2[U, U] R \subseteq U$.

This remark will be freely used in the whole paper without specific mention.

We begin with the following lemmas which are essential for developing the proof of our results.

Lemma 2.1 ([5], Lemma 4). Let $R$ be a prime ring of characteristic different from 2 and $U \nsubseteq$ $\nsubseteq Z(R)$ be a Lie ideal of $R$ and if $a U b=\{0\}$, then $a=0$ or $b=0$.

Lemma 2.2 ([3], Theorem 4). Let $R$ be a prime ring of characteristic different from 2 and $U$ be a square closed Lie ideal of $R$. If $d: R \rightarrow R$ is an additive mapping such that $d\left(x^{2}\right)=2 x d(x)$ for all $x \in U$, then $d(x y)=x d(y)+y d(x)$ for all $x, y \in U$.

Lemma 2.3 ([1], Proposition 2.10). Let $R$ be a prime ring and $F: R \rightarrow R_{C}$ be an additive mapping satisfying $F(r s)=r F(s)$ for all $r, s \in R$. Then there exists $q \in Q_{l}\left(R_{C}\right)$ such that $F(r)=r q$ for all $r \in R$.

Lemma 2.4 ([10], Lemma 3.3). Let $R$ be a 2-torsion free semiprime ring and $U$ be a nonzero Lie ideal of $R$. If $[U, U] \subseteq Z(R)$, then $U \subseteq Z(R)$.

Lemma 2.5 ([15], Lemma 2.7). Let $G_{1}, G_{2}, \ldots, G_{n}$ be additive groups, $R$ a 2-torsion free semiprime ring and $U \nsubseteq Z(R)$ be a Lie ideal of $R$. Suppose that mappings $S: G_{1} \times G_{2} \times \ldots \times G_{n} \rightarrow R$ and $T: G_{1} \times G_{2} \times \ldots \times G_{n} \rightarrow R$ are additive in each argument. If $S\left(a_{1}, a_{2}, \ldots, a_{n}\right) x T\left(a_{1}, a_{2}, \ldots, a_{n}\right)=$ $=0$ for all $x \in U, a_{i} \in G_{i}, \quad i=1,2, \ldots, n$, then $S\left(a_{1}, a_{2}, \ldots, a_{n}\right) x T\left(b_{1}, b_{2}, \ldots, b_{n}\right)=0$ for all $x \in U, a_{i}, b_{i} \in G_{i}, i=1,2, \ldots, n$.

3. Generalized Jordan left derivation on prime ring. We begin with the following lemmas which are essential for developing the proof of our results.

Lemma 3.1. Let $R$ be a 2-torsion free ring and $U$ be a square closed Lie ideal of $R$. Suppose that $F: R \rightarrow R$ be a generalized Jordan left derivation with associated Jordan left derivation $d: R \rightarrow R$ on $U$. Then

(i) $F(x y+y x)=x F(y)+y F(x)+x d(y)+y d(x)$ for all $x, y \in U$,

(ii) $F(x y x)=x y F(x)+2 x y d(x)+x^{2} d(y)-y x d(x)$ for all $x, y \in U$,

(iii) $F(x y z+z y x)=x y F(z)+z y F(x)+2 x y d(z)+2 z y d(x)+x z d(y)+z x d(y)-y x d(z)-y z d(x)$ for all $x, y, z \in U$.

Proof. (i) Since $F$ is a generalized Jordan left derivation on $U$, we have $F\left(x^{2}\right)=x F(x)+x d(x)$ for all $x \in U$. Compute $W=F\left((x+y)^{2}\right)$ in two different ways. On one hand we find that $W=F\left((x+y)^{2}\right)=x F(x)+x F(y)+y F(x)+y F(y)+x d(x)+x d(y)+y d(x)+y d(y)$ for all $x, y \in U$. On the other hand

$$
\begin{gathered}
F\left((x+y)^{2}\right)=F\left(x^{2}+y^{2}+x y+y x\right)= \\
=x F(x)+x d(x)+y F(y)+y d(y)+F(x y+y x) \quad \text { for all } \quad x, y \in U .
\end{gathered}
$$


Comparing two expressions, we obtain the required result.

(ii) Replacing $y$ by $x y+y x$ in (i), we get

$$
\begin{gathered}
F(x(x y+y x)+(x y+y x) x)=x F(x y+y x)+(x y+y x) F(x)+ \\
+x d(x y+y x)+(x y+y x) d(x) \quad \text { for all } \quad x, y \in U .
\end{gathered}
$$

Since $d: R \rightarrow R$ is a Jordan left derivation, linearizing $d\left(x^{2}\right)=2 x d(x)$, we find that

$$
d(x y+y x)=x d(y)+y d(x)+x d(y)+y d(x) \quad \text { for all } \quad x, y \in U,
$$

and hence,

$$
\begin{aligned}
F(x(x y+y x)+ & (x y+y x) x)=x^{2} F(y)+2 x y F(x)+4 x y d(x)+3 x^{2} d(y)+ \\
& +y x d(x)+y x F(x) \quad \text { for all } \quad x, y \in U .
\end{aligned}
$$

On the other hand, we have

$$
\begin{gathered}
F(x(x y+y x)+(x y+y x) x)=F\left(x^{2} y+y x^{2}\right)+2 F(x y x)= \\
=x^{2} F(y)+y x F(x)+3 y x d(x)+x^{2} d(y)+2 F(x y x) \quad \text { for all } \quad x, y \in U .
\end{gathered}
$$

Combining (3.1) and (3.2) and using the fact that $R$ is 2-torsion free, we obtain

$$
F(x y x)=x y F(x)+x^{2} d(y)+2 x y d(x)-y x d(x) \quad \text { for all } \quad x, y \in U .
$$

(iii) Replace $x$ by $x+z$ in (ii), to get

$$
\begin{gathered}
F((x+z) y(x+z))=x y F(x)+x y F(z)+z y F(x)+z y F(z)+2 x y d(x)+ \\
+2 x y d(z)+2 z y d(x)+2 z y d(z)+x^{2} d(y)+ \\
+x z d(y)+z x d(y)+z^{2} d(y)-y x d(x)-y x d(z)- \\
-y z d(x)-y z d(z) \quad \text { for all } \quad x, y, z \in U .
\end{gathered}
$$

On the other hand, we have

$$
\begin{gathered}
F((x+z) y(x+z))=F(x y x)+F(z y z)+F(x y z+z y x)= \\
=x y F(x)+x^{2} d(y)+2 x y d(x)-y x d(x)+z y F(z)+ \\
+z^{2} d(y)+2 z y d(z)-y z d(z)+F(x y z+z y x) .
\end{gathered}
$$

Now, comparing (3.3) and (3.4), we get (iii).

Lemma 3.2. Let $R$ be a prime ring of characteristic different from 2 and $U$ be a noncommutative square closed Lie ideal of $R$. If $T: R \rightarrow R$ is an additive mapping which satisfies the condition $T\left(x^{2}\right)=T(x) x$ for all $x \in U$, then $T(x y)=T(x) y$ for all $x, y \in U$. 
Proof. We have

$$
T\left(x^{2}\right)=T(x) x \quad \text { for all } \quad x \in U
$$

Linearizing (3.5), we get

$$
T(x y+y x)=T(x) y+T(y) x \quad \text { for all } \quad x, y \in U .
$$

Replacing $y$ by $x y+y x$ in (3.6), we obtain

$$
T(x(x y+y x)+(x y+y x) x)=T(x) x y+T(x) y x+T(x) y x+T(y) x^{2} \quad \text { for all } \quad x, y \in U .
$$

On the other hand

$$
\begin{gathered}
T(x(x y+y x)+(x y+y x) x)=T(2 x y x)+T\left(x^{2} y+y x^{2}\right)= \\
\quad=2 T(x y x)+T(x) x y+T(y) x^{2} \quad \text { for all } \quad x, y \in U .
\end{gathered}
$$

Now, comparing (3.7) and (3.8), we have

$$
T(x y x)=T(x) y x \quad \text { for all } \quad x, y \in U .
$$

Again, replace $x$ by $x+z$ in (3.9), to get

$$
T(x y z+z y x)=T(x) y z+T(z) y x \quad \text { for all } \quad x, y, z \in U .
$$

Let us consider

$$
W=T(x y z y x+y x z x y) \quad \text { for all } \quad x, y, z \in U .
$$

This can be rewritten as $W=T(x(y z y) x)+T(y(x z x) y)$ for all $x, y, z \in U$. Using (3.9) in the above relation, we obtain

$$
W=T(x) y z y x+T(y) x z x y \quad \text { for all } \quad x, y, z \in U .
$$

Again, we can write $W=T((x y) z(y x)+(y x) z(x y))$ for all $x, y, z \in U$, and hence by application of (3.10), we find that

$$
W=T(x y) z y x+T(y x) z x y \quad \text { for all } \quad x, y, z \in U .
$$

Equating (3.12) and (3.13), we get $\{T(x y)-T(x) y\} z y x+\{T(y x)-T(y) x\} z x y=0$ for all $x, y, z \in U$. Now, we introduce a biadditive mapping $B(x, y)=T(x y)-T(x) y$ for all $x, y \in U$. Using this in the above relation, we obtain $B(x, y) z y x+B(y, x) z x y=0$ for all $x, y, z \in U$. Using relation (3.6), we can say that $B(x, y)=-B(y, x)$. Hence we get $B(x, y) z[x, y]=0$ for all $x, y, z \in U$. Hence, by Lemma 2.5 , we have $B(x, y) z[u, v]=0$ for all $x, y, z, u, v \in U$, that is, $B(x, y) U[u, v]=\{0\}$ for all $x, y, u, v \in U$. Since $U$ is a noncommutative Lie ideal of $R$ and by Lemma 2.1, we find $B(x, y)=0$ for all $x, y \in U$ i.e., $T(x y)=T(x) y$ for all $x, y \in U$.

By similar arguments as the above with necessary variations, we can prove the following lemma.

Lemma 3.3. Let $R$ be a prime ring of characteristic different from 2 and $U$ be a noncommutative square closed Lie ideal of $R$. If $T: R \rightarrow R$ is an additive mapping which satisfies the condition $T\left(x^{2}\right)=x T(x)$ for all $x \in U$, then $T(x y)=x T(y)$ for all $x, y \in U$. 
Theorem 3.1. Let $R$ be a prime ring of characteristic different from 2 and $U$ be a noncommutative square closed Lie ideal of $R$. If $R$ admits a generalized left derivation $F: R \rightarrow R$ with associated Jordan left derivation $d: R \rightarrow R$ on $U$, then $d=0$ on $R$.

Proof. Since $F$ is a generalized left derivation on $U$, we have $F(x y)=x F(y)+y d(x)$ for all $x, y \in U$. Replacing $x$ by $x^{2}$ in the above expression, we get $F\left(x^{2} y\right)=x^{2} F(y)+y d\left(x^{2}\right)$ for all $x, y \in U$. Since $d: R \rightarrow R$ is a Jordan left derivation, we find that

$$
F\left(x^{2} y\right)=x^{2} F(y)+2 y x d(x) \quad \text { for all } \quad x, y \in U
$$

On the other hand,

$$
F\left(x^{2} y\right)=F(x(x y))=x F(x y)+x y d(x)=x^{2} F(y)+2 x y d(x) .
$$

Combining (3.14) and (3.15) and using the fact that char $R \neq 2$, we obtain $[x, y] d(x)=0$ for all $x, y \in U$. Now, replacing $y$ by $2 y z$, we find that $[x, y] z d(x)=0$ for all $x, y, z \in U$. Hence, by Lemma 2.1, for each $x \in U$ either $[x, y]=0$ or $d(x)=0$. Now, for each $x \in U$, let $A=\{x \in$ $\in U \mid[x, y]=0$ for all $y \in U\}$ and $A^{\prime}=\{x \in U \mid d(x)=0\}$. Moreover, $U$ is the set-theoretic union of $A$ and $A^{\prime}$. But a group cannot be the set-theoretic union of two proper subgroups. Hence, by Brauer's trick, we have either $U=A$ or $U=A^{\prime}$. In the former case, we have $[x, y]=0$ for all $x, y \in U$, a contradiction. In the latter case, we have $d(x)=0$ for all $x \in U$. Now, replace $x$ by $2 r[t, x]$ and using char $R \neq 2$, to get $r d([t, x])+[t, x] d(r)=0$ for all $t, x \in U$ and $r \in R$. This implies that $[t, x] d(r)=0$ for all $t, x \in U$ and $r \in R$. Again, replacing $x$ by $2 x y$ and using the fact that $\operatorname{char} R \neq 2$, we get $[t, x] y d(r)=0$ for all $t, x, y \in U$ and $r \in R$, that is, $[t, x] U d(r)=\{0\}$ for all $t, x \in U$ and $r \in R$, thus by the application of Lemma 2.1 yields that either $d(r)=0$ for all $r \in R$ or $[t, x]=0$ for all $t, x \in U$. Hence, $d=0$ on $R$, since $U$ is noncommutative Lie ideal of $R$.

Theorem 3.2. Let $R$ be a prime ring of characteristic different from 2 and $U$ be a noncommutative square closed Lie ideal of $R$. Suppose $F: R \rightarrow R$ is a generalized left derivation with associated Jordan left derivation $d: R \rightarrow R$ on $U$. Then every generalized Jordan left derivation on $U$ is a generalized left derivation on $U$.

Proof. If associated Jordan left derivation $d=0$, then $F$ is a Jordan right centralizer on $U$. Therefore, in view of Lemma 3.2, $F$ is a right centralizer on $U$. Hence for $d=0$, it is generalized left derivation on $U$.

Suppose that associated Jordan left derivation $d \neq 0$ on $U$. Thus, in view of Lemma 2.2, $d$ is a left derivation on $U$. Since $F, d$ and $T$ are additive maps on $U$, we write $T=F-d$. Then we find that

$$
\begin{aligned}
T\left(x^{2}\right) & =(F-d)\left(x^{2}\right)=F\left(x^{2}\right)-d\left(x^{2}\right)=x F(x)+x d(x)-x d(x)-x d(x)= \\
& =x F(x)-x d(x)=x(F(x)-d(x))=x T(x) \quad \text { for all } \quad x \in U
\end{aligned}
$$

This implies that $T\left(x^{2}\right)=x T(x)$ for all $x \in U$, that is, $T$ is a Jordan right centralizer on $U$. Thus, by Lemma 3.3, one can conclude that $T$ is a right centralizer on $U$. Therefore, we prove that $F$ can be written as $F=d+T$, where $d$ is a left derivation and $T$ is a right centralizer on $U$. This completes the proof of the theorem.

As immediate consequences of the above theorems, we have the following corollaries. 
Corollary 3.1. Let $R$ be a prime ring of characteristic different from 2 and $U$ be a square closed Lie ideal of $R$. If $R$ admits a nonzero Jordan left derivation $d: R \rightarrow R$, then $U$ is a commutative Lie ideal.

Corollary 3.2. Let $R$ be a noncommutative prime ring of characteristic different from 2 . If $R$ admits a generalized Jordan left derivation $F: R \rightarrow R$ with associated Jordan left derivation $d: R \rightarrow R$, then $F(x)=x q$ for all $x \in R$ and $q \in Q_{l}\left(R_{C}\right)$.

Proof. Since $R$ is a noncommutative prime ring of characteristic different from two and $F$ be a generalized Jordan left derivation with associated Jordan left derivation $d$ on $R$. Thus in view of Theorems 3.2 and 3.1, we have $d=0$ on $R$. Thus, we obtained $F\left(x^{2}\right)=x F(x)$ for all $x \in R$ that is, $F$ is Jordan right centralizer and therefore, in view of Lemma 3.3, $F$ is right centralizer on $R$ i.e., $F(x y)=x F(y)$ for all $x, y \in R$. Hence by Lemma 2.3, there exists $q \in Q_{l}\left(R_{C}\right)$ such that $F(x)=x q$ for all $x \in R$.

In conclusion, it is tempting to conjecture as follows:

Conjecture 3.1. Let $R$ be a 2-torsion free semiprime ring and $U$ be a Lie ideal of $R$. If $F: R \rightarrow R$ is a generalized Jordan left derivation on $U$, then $F$ is a generalized left derivation on $U$.

4. Generalized left biderivation on prime ring. Over the last few decades, several authors have investigated the relationship between the commutativity of a ring $R$ and certain specific types of derivations of $R$. A classical result due to Posner who proved that if a prime ring $R$ admits a nonzero derivation $d$ such that $[d(x), x] \in Z(R)$ for all $x \in R$, then $R$ is commutative. Then many authors generalized this result in the setting of left ideal, Lie ideal etc. in prime and semiprime ring. In [8], Bresar and Vukman shown that a prime ring must be commutative if it admits a nonzero left derivation. In [1], Shakir obtained that every generalized left biderivation $G: R \times R \rightarrow R$ with associated left biderivation $B: R \times R \rightarrow R$ on a prime ring of char $R \neq 2$ is either a right bicentralizer on $R$ or $R$ is commutative. In this section, our goal is to extend the above mentioned result in the setting of generalized left biderivation on Lie ideal. The main result stated as follows:

Theorem 4.1. Let $R$ be prime ring of $\operatorname{char} R \neq 2$ and $U$ be a square closed Lie ideal of $R$. Suppose $R$ admit biadditive maps $G, B: R \times R \rightarrow R$ such that $G(x y, z)=x G(y, z)+y B(x, z)$ for all $x, y, z \in U$, where $B(x y, z)=x B(y, z)+y B(x, z)$ for all $x, y, z \in U$. Then either $U \subseteq Z(R)$ or $G$ is right bicentralizer on $U$.

Proof. We are given that $G$ is a generalized left biderivation, we have

$$
G(x y, z)=x G(y, z)+y B(x, z) \quad \text { for all } \quad x, y, z \in U .
$$

Replacing $y$ by $2 y w$ in (4.1) and using the fact that char $R \neq 2$, we find that

$$
\begin{gathered}
G(x(y w), z)=x G(y w, z)+y w B(x, z)= \\
=x y G(w, z)+x w B(y, z)+y w B(x, z) \text { for all } \quad x, y, z, w \in U .
\end{gathered}
$$

On the other hand, we obtain

$$
\begin{gathered}
G((x y) w, z)=x y G(w, z)+w B(x y, z)= \\
=x y G(w, z)+w x B(y, z)+w y B(x, z) \quad \text { for all } \quad x, y, z, w \in U .
\end{gathered}
$$


Now, combining (4.2) and (4.3), we have

$$
[x, w] B(y, z)+[y, w] B(x, z)=0 \quad \text { for all } \quad x, y, z, w \in U .
$$

Replace $y$ by $w$ in (4.4), to get

$$
[x, w] B(w, z)=0 \quad \text { for all } \quad x, z, w \in U .
$$

Again, replacing $w$ by $2 w t$ and using char $R \neq 2$ in the above relation, we obtain

$$
[x, w] t B(w, z)=0 \quad \text { for all } \quad x, z, w, t \in U .
$$

Then, by Lemma 2.1, we get for each $w \in U$, either $[x, w]=0$ or $B(w, z)=0$ for all $x, z \in U$. Let

$$
A=\{w \in U \mid[x, w]=0 \text { for all } x \in U\} \quad \text { and } \quad A^{\prime}=\{w \in U \mid B(w, z)=0 \text { for all } z \in U\} .
$$

Then obviously, $A$ and $A^{\prime}$ are the additive subgroups of $U$ and $A \cup A^{\prime}=U$. Therefore, by Brauer's trick, we have either $U=A$ or $A^{\prime}=U$. If $U=A$, then $[x, w]=0$ for all $x, w \in U$, then by Lemma 2.4, $U \subseteq Z(R)$ and if $B(w, z)=0$ for all $w, z \in U$, then $G$ is a right bicentralizer on $U$.

Hence, we get the required result.

1. Ali S. On generalized left derivations in rings and Banach algebras // Aequat. math. - 2011. - 81. - P. $209-226$.

2. Ashraf M., Ali S. On generalized Jordan left derivations in rings // Bull. Korean Math. Soc. - 2008. - 45, № 2. P. $253-261$.

3. Ashraf M., Rehman N. On Lie ideals and Jordan left derivations of prime rings // Arch. Math. (Brno). - 2000. - 36. P. $201-206$.

4. Ashraf M., Rehman N., Ali S. On Jordan left derivations of Lie ideals in prime rings // Suouth Asian Bull. Math. 2001. - 25. - P. $379-382$.

5. Bergen J., Herstein I. N., Kerr J. W. Lie ideals and derivations of prime rings // J. Algebra. - 1981. - 71 - - P. 259 - 267.

6. Beidar K. I., Martinedale III W. S., Mikhaleu A. V. Ring with generalized identities // Monographs and Textbooks in Pure and Appl. Math. - New York: Markel Dekker, 1995.

7. Bresar M. On generalized bi-derivation and related maps // J. Algebra. - 1995. - 172. - P. $764-786$.

8. Bresar M., Vukman J. On left derivations and related mappings // Proc. Amer. Math. Soc. - 1990. - 110. - P. 7 - 16.

9. Deng $Q$. On Jordan left derivations // Math. J. Okayama Univ. - 1992. - 34. - P. 145-147.

10. Herstein I. N. On the Lie structure of associative rings // J. Algebra. - 1970. - 14, № 4. - P. $561-571$.

11. Herstein I. N. Topics in ring theory. - Chicago: Univ. Chicago Press, 1969.

12. Jun K. W., Kim B. D. A note on Jordan left derivations // Bull. Korean Math. Soc. - 1996. - 33, № 2. - P. $221-228$.

13. Martindale III W. S. Prime ring satisfying a generalized polynomial identity // J. Algebra. - 1969. - 12. - P. 576 - 584.

14. Muthana N. M. Left centralizer traces, generalized biderivations, left bi-multipliers and generalized Jordan biderivations // Aligarh Bull. Math. - 2007. - 26, № 2. - P. 33 -45.

15. Rehman N., Hongan M. Generalized Jordan derivations on Lie ideals associate with Hochschild 2-cocycles of rings // Rend. Circ. mat. Palermo. - 2011. - 60(2), № 3. - P. 437-444.

16. Vukman J. Jordan left derivations on semiprime rings // Math. J. Okayama Univ. - 1997. - 39. - P. 1-6.

17. Zaidi S. M. A., Ashraf M., Ali S. On Jordan ideals and left $(\theta, \theta)$-derivation in prime rings // Int. J. Math. and Math. Sci. - 2004. - 37. - P. $1957-1965$.

18. Zalar B. On centralizers of semiprime rings // Comment. math. Univ. carol. - 1991. - 32, № 4. - P. $609-614$. 